\title{
Ultra-peripheral heavy-ion collisions with the CMS experiment
}

\author{
The CMS Collaboration
}

\begin{abstract}
Ultraperipheral collisions (UPCs) of heavy ions involve long range electromagnetic interactions at impact parameters larger than sum of their radii where hadronic interaction is largely suppressed and the exchanged photon materializes into qq(bar) bound state after interacting with the gluonic field of the target proton or ion. Photoproduction of heavy vector mesons (J/psi, Upsilon) thus provide direct information on the gluon distribution functions in the nucleon/nucleus at very low values of Bjorken-x. The CMS experiment has excellent capabilities for the measurement of the heavy vector mesons in the dimuon decay channel using the tracker and the muon chambers. The measured coherent $\mathrm{J} / \mathrm{psi}$ photoproduction cross section in ultraperipheral $\mathrm{Pb}-\mathrm{Pb}$ collisions using 2011 $\mathrm{PbPb}$ data and Upsilon photoproduction in ultraperipheral $\mathrm{pPb}$ collisions during 2013, will be presented. The prospects for future measurements using the data collected in the $2015 \mathrm{PbPb}$ run will also be described.
\end{abstract}

\section{Introduction}

\subsection{Coherent $J / \psi$ production in PbPb UPCs}

Ultraperipheral collisions (UPCs) of protons and ions involve electromagnetic interactions at impact parameters larger than the sum of the radii of colliding nuclei. These photon-nucleus and photonphoton collisions are produced at the LHC in abundance [1][2]. The cross section for photon-nucleus collisions is proportional to $Z^{2}$, where $Z$ is the charge of the nucleus. The cross section for producing vector mesons such as $J / \psi, \psi(2 S)$ and $\Upsilon$ is proportional to the square of the nuclear gluon density [3][4]. the gluon distribution function within nuclei is poorly constrained, especially at low Bijorken- $x$. This is currently a subject of strong interest since it dominates the initial state of heavy ion collisions. Photoproduction of vector mesons has been studied with lepton beams [5] and later at the electron-proton collider HERA [6][7]. The STAR and PHENIX collaborations at RHIC have studied $\rho^{0}$ and $J / \psi$ production in ultraperipheral AuAu collisions at $\sqrt{s_{N N}}=200 \mathrm{GeV}$ [8][9]. These studies were not able to considerably constrain the nuclear gluon distribution function due to the small sample size. The production rate for UPC physics processes is much higher at the LHC. By measuring coherent $J / \psi$ photoproduction in ultraperipheral $\mathrm{PbPb}$ collisions at $\sqrt{s_{N N}}=2.76 \mathrm{TeV}$, the ALICE collaboration has found evidence that the nuclear gluon density is below that expected for a simple superposition of protons and neutrons in the nucleus[10][11].

\subsection{Exclusive $\Upsilon$ production in pPb UPCs}

In UPCs, hadrons (ions) interact electromagnetically where an exchanged (quasi)real photon materializes into a $q \bar{q}$ bound state after interacting with the gluon field of the target proton (ion) [2]. Since the 
Figure 1. Diagrams representing exclusive $\Upsilon$ photoproduction (left), and exclusive dimuon $\mathrm{QED}$ continuum (right) in $\mathrm{pPb}$ collisions.
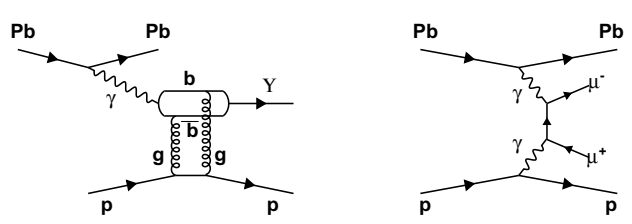

incoming hadrons remain intact after the interaction and no other particles are produced in the event, the process is called "exclusive". Given that the photon flux scales with the square of the emitting charge $\left(Z^{2}\right)$, the emission of quarireal photons from the $\mathrm{Pb}$-ion is strongly enhanced compared to that from the proton. Figure 1 (left) shows the diagram for exclusive $\Upsilon$ photoproduction in $\mathrm{pPb}$ collisions, dominated by the process $\mathrm{pPb} \rightarrow(\gamma \mathrm{p}) \rightarrow \mathrm{p} \Upsilon(\mathrm{nS}) \mathrm{Pb}$. Similarly, the exchanged photon can interact with another equivalently radiated photon from the other hadron leading to two-photon collisions followed by exclusive dilepton production as shown in Fig. 1 (right) [2][12]. Since we are interested in studying exclusive $\Upsilon$ production via its dilepton decay, such photon-photon continuum constitutes a non-irreducible background for our signal.

\section{Results}

\subsection{Coherent $J / \psi$ photoproduction in ultra-peripheral $\mathrm{PbPb}$ collisions at $\sqrt{s_{N N}}=2.76 \mathrm{TeV}$}

The coherent $J / \psi$ photoproduction cross section in ultraperipheral $\mathrm{PbPb}$ collisions at $\sqrt{s_{N N}}=2.76$ $\mathrm{TeV}$, in conjunction with at least one neutron on the side of the interaction point and no neutron activity on the other side, is measured to be $d \sigma_{X_{n} O n}^{c o h} / d y(J / \psi)=0.36 \pm 0.04$ (stat) \pm 0.04 (syst) mb in the rapidity interval $1.8<|y|<2.3$. This measurement is extrapolated to the total coherent $J / \psi$ cross section, resulting in $d \sigma^{c o h} / d y(J / \psi)=1.82 \pm 0.22$ (stat) \pm 0.20 (syst) \pm 0.19 (theo) $\mathrm{mb}$ in the measured rapidity interval. These results complement recent measurements on coherent $J / \psi$ photoproduction in ultraperipheral $\mathrm{PbPb}$ collisions at $\sqrt{s_{N N}}=2.76 \mathrm{TeV}$ by the ALICE collaboration. An impulse approximation model prediction is strongly disfavored, indicating that nuclear effects expected to be present at low $x$ and $Q^{2}$ values are needed to describe the data. The prediction given by the leading twist approximation, which includes nuclear gluon shadowing, is consistent with the data.

\subsection{Exclusive $\Upsilon$ photoproduction in ultra-peripheral pPb collisions at $\sqrt{s_{N N}}=5.02 \mathrm{TeV}$}

We reported the first measurement of the exclusive photoproduction of $\Upsilon(1 \mathrm{~S}, 2 \mathrm{~S}, 3 \mathrm{~S})$ mesons in the $\mu^{+} \mu^{-}$decay modes in ultraperipheral $\mathrm{pPb}$ collisions at $\sqrt{s_{N N}}=5.02 \mathrm{TeV}$, using data collected with the CMS detector in 2013, corresponding to an integrated luminosity of 32.6 inverse nb. The exclusive photoproduction cross sections have been measured as a function of the photon-proton center of mass energy in the range $91<W_{\gamma p}<826 \mathrm{GeV}$, probing the region of parton fractional momenta in the proton $x \approx 10^{-4}-10^{-2}$, bridging a previously unexplored region between HERA and LHCb measurements. Our data are compatible with a power law dependence of $\sigma\left(W_{\gamma}\right)$, disfavouring faster rising extrapolations of HERA results (STARLIGHT) and LO pQCD predictions. The differential cross section $d \sigma / d|t|$, has been also measured in the range $|t|<1.0 \mathrm{GeV}^{2}$, and the exponential spectral slope $b=4.5 \pm 1.7$ (stat) \pm 0.6 (syst) $\mathrm{GeV}^{-2}$ has been extracted, in agreement with earlier measurements and consistent with predictions based on pQCD models. The data presented here provide new constraints for the evolution of the gluon density at low values of fractional momenta $x$ in the proton. 

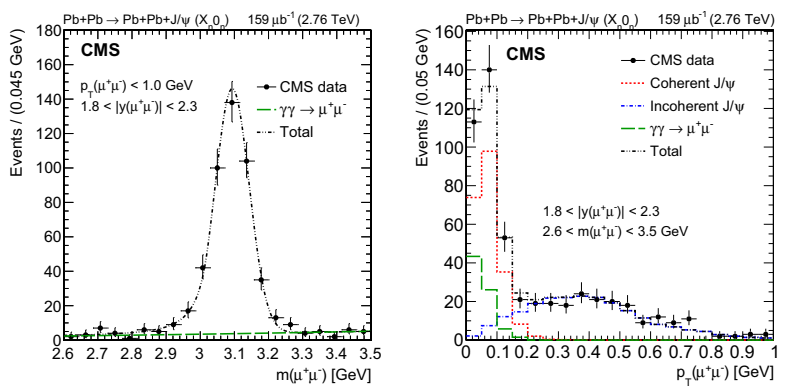

Figure 2. Results from the simultaneous fit to dimuon invariant mass (left) and $p_{T}$ (right) distributions from opposite-sign muon pairs with $p_{T}<10 \mathrm{GeV}, 1.8<|y|<2.3$ and $2.6<m\left(\mu^{+} \mu^{-}\right)<3.5 \mathrm{GeV}$ for the $X_{n} O_{n}$ break-up mode, after all selections are applied. In the left panel the green curve represents the $\gamma+\gamma$ component (secondorder polynomial) and the black curve the sum of the $\gamma+\gamma$, coherent $J / \psi$, and incoherent $J / \psi$ components. In the right panel the green, red, and blue curves represent $\gamma+\gamma$, coherent $J / \psi$ and incoherent $J / \psi$ conponents. Only statistical uncertaintied are shown. The data are not corrected by acceptance and efficiencies, and the MC templates are folded with the detector response simulation.
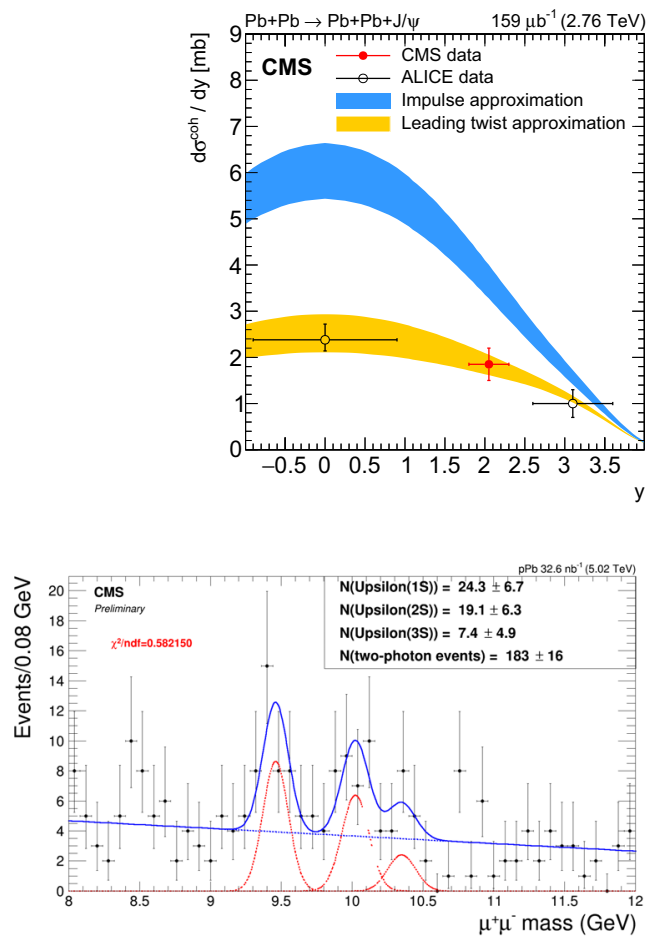

Figure 3. Differential cross section versus rapidity for coherent $J / \psi$ production in ultraperipheral $\mathrm{PbPb}$ collisions at $\sqrt{s_{N N}}=2.76 \mathrm{TeV}$, measured by ALICE[2] and CMS. the vertical error bars include the statistical and systematic uncertainties added in quadrature, and the horizontal bars represent the range of the measurements in $y$. Also the impulse approximation and the leading twist approximation calculations are shown.

Figure 4. Invariant mass distribution for the $\mu^{+} \mu^{-}$pairs in the range $8<M_{\mu^{+} \mu^{-}}<12 \mathrm{GeV}$. The three peaks correspond to the $\Upsilon(1 S), \Upsilon(2 S)$, and $\Upsilon(3 S)$ mesons. The fit to the data is performed with ROOFIT. The blue line corresponds to the polynomial fit to the two-photon QED continuum, the red dashed line to the Gaussian fit of the resonances. The corresponding number of events are indicated in the legend. 
Figure 5. Distributions of the transverse momentum squared $p_{T}^{2}$ and rapidity $y$ of the muon pairs with invariant mass $9.12<$ $m_{\mu^{+} \mu^{-}}<10.64 \mathrm{GeV}$ in the conbined $\mathrm{pPb}$ and $\mathrm{Pbp}$ samples.
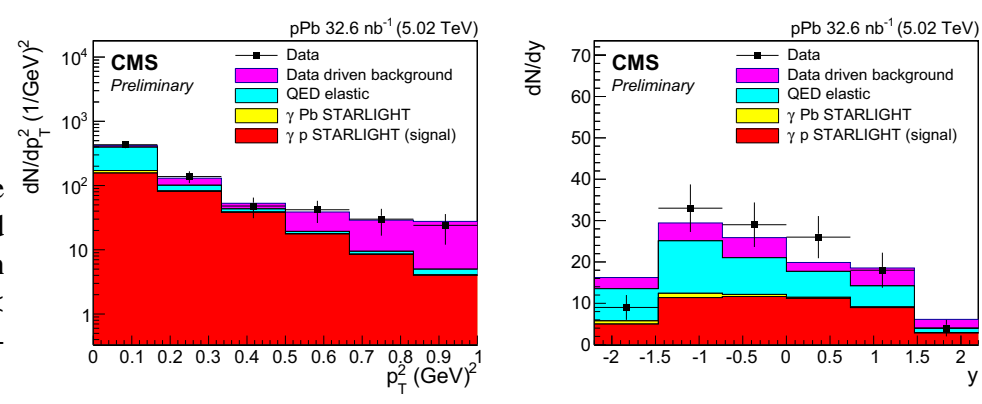

Figure 6. Cross section for exclusive $\Upsilon(1 \mathrm{~S})$ photoproduction, $\gamma p \rightarrow \Upsilon(1 \mathrm{~S}) p$ as a function of photon-proton center-of-mass energy.

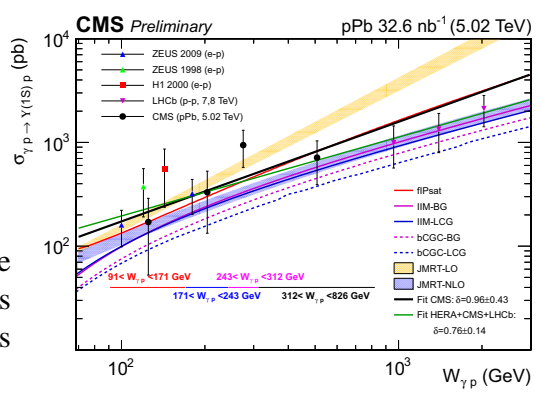

\section{References}

[1] C. A. Bertulani, S. R. Klein, and J. Nystrand, "Physics of ultra-peripheral nuclear collisions" Annual Review of Nuclear and Particle science 55, no. 1, 271-310 (2005)

[2] A. Baltz et al., "The physics of ultraperipheral collisions at the LHC" Physics Reports 458, no. 13, 1-171 (2008)

[3] M. Ryskin, "Diffractive $J / \psi$ electroproduction in LLA QCD" Z. Phys. C57, 89-92 (1993)

[4] S. J. Brodsky et al., "Diffractive leptoproduction of vector mesons in QCD" Phys. Rev. D50, 3134-3144 (1994)

[5] L. J. Lanzerotti et al., "High-Energy Photoproduction of Neutral Rho Mesons" Phys. Rev. Lett. 15, 210-213 (1965)

[6] ZEUS Collaboration, "Exclusive photoproduction of $J / \psi$ mesons at HERA" Eur.Phys.J. C24, 345-360 (2002)

[7] ZEUS Collaboration, "Exclusive electroproduction of $J / \psi$ mesons at HERA" Nucl.Phys. B695, 3-37 (2004)

[8] STAR Collaboration, " $\rho^{0}$ photoproduction in ultraperipheral relativistic heavy ion collisions at $\sqrt{s_{N N}}=200 \mathrm{GeV}$ " Phys. Rev. C 77, 034910 (2008)

[9] S. Afanasiev et al., "Photoproduction of and of high mass in ultra-peripheral $\mathrm{Au}+\mathrm{Au}$ collisions at $\sqrt{s_{N N}}=200 \mathrm{GeV} "$ Phys. Lett. B 679, no. 4, 321-329 (2009)

[10] ALICE Collaboration, "Coherent $J / \psi$ photoproduction in ultra-peripheral $\mathrm{Pb}-\mathrm{Pb}$ collisions at $\sqrt{s_{N N}}=2.76$ TeV" Phys. Lett. B718, 1273-1283 (2013)

[11] V. Guzey, E. Kryshen, M. Strikman, and M. Zhalov, "Evidence for nuclear gluon shadowing from the ALICE measurements of $\mathrm{PbPb}$ ultraperipheral exclusive $J / \psi$ production" Phys.Lett. B726, 290-295 (2013)

[12] V. Budnev et al., "The Two photon particle production mechanism. Physical problems, Applications, Equivalent photon approximation" Phys. Rep. 15. 181 (1975) 